\title{
46. DATA REPORT: ROCK MAGNETIC PROPERTIES OF HYDROTHERMALLY ALTERED SEDIMENTS FROM MIDDLE VALLEY ${ }^{1}$
}

\author{
H. Oda ${ }^{2}$ and U. Körner ${ }^{3}$
}

\begin{abstract}
Rock magnetic studies on sediment samples from Ocean Drilling Program Sites 855 and 857, Middle Valley, Juan de Fuca Ridge, were carried out during Leg 139. Results of natural remanent magnetization intensity and susceptibility show that the concentration of magnetic minerals is high. The thermomagnetic analyses and the thermal demagnetization of composite isothermal remanent magnetizations (Lowrie, 1990) were also conducted.
\end{abstract}

\section{INTRODUCTION}

Magnetic minerals in sediments change their magnetic properties through diagenesis and alteration after sedimentation (King and Channell, 1991). In anoxic conditions, magnetic minerals such as titanomagnetite or magnetite tend to be dissolved with increasing depth. There have been several studies of dissolution of magnetic minerals in anoxic sediments from continental margins (Karlin, 1990; Channell and Hawthorne, 1990; Torii et al., 1992).

Leg 139 operated in Middle Valley, northern Juan de Fuca Ridge, to investigate hydrothermal processes and products at a sedimentcovered seafloor spreading axis. Middle Valley is presently an active hydrothermal area providing a good opportunity to investigate the effects of pervasive hydrothermal alteration on magnetic minerals in sediments.

Rock magnetic studies were conducted on paleomagnetic samples collected during the cruise. We measured natural remanent magnetizations, susceptibilities, and hysteresis parameters. We also conducted thermomagnetic analyses and thermal demagnetizations of composite isothermal remanent magnetizations (IRMs) (Lowrie, 1990).

\section{SAMPLES}

During Leg 139, holes were drilled at four sites to characterize distinct hydrologic environments within the sedimented rift valley (Fig. 1). Sediments were mainly terrigenous or hemipelagic turbidites. Oriented paleomagnetic cube samples $\left(2.1 \times 2.1 \times 1.3 \mathrm{~cm}^{3}\right)$ were taken from silty to clayey parts of the sediment cores at Sites 855 and 857 .

Samples from Site 857 were used exclusively for this rock magnetic study, because this site was drilled over a hydrothermal reservoir (Davis, Mottl, Fisher, et al., 1992) and recovered a continuous sedimentary section of about $470 \mathrm{~m}$. Site 857 is located $5.2 \mathrm{~km}$ west of the normal fault scarp that forms the eastern boundary of Middle Valley, and about $1.5 \mathrm{~km}$ east of the sediment-buried fault that forms the current structural boundary of the central part of the rift (Fig. 1). Four holes were drilled at Site 857 and the sediment samples for paleomagnetic measurements were collected from Holes $857 \mathrm{~A}$ and $857 \mathrm{C}$. Hole $857 \mathrm{~A}$ was drilled by advanced piston core (APC) coring down to $85 \mathrm{mbsf}$, and continued with extended core barrel (XCB) coring to $111 \mathrm{mbsf}$, Hole $857 \mathrm{C}$ was drilled by rotary core barrel (RCB) coring to $561 \mathrm{mbsf}$, and at $471 \mathrm{mbsf}$ an altered basaltic sill was encountered. Estimated temperatures in the hole gradually increased

\footnotetext{
${ }^{1}$ Mottl, M.J., Davis, E.E., Fisher, A.T., and Slack, J.F. (Eds.), 1994. Proc. ODP, Sci. Results, 139: College Station, TX (Ocean Drilling Program).

${ }_{3}^{2}$ Department of Geology and Mineralogy, Kyoto University, Kyoto 606-1, Japan.

${ }^{3}$ Institut furr Allgemeine und Angewandte Geophysik, Theresienstrasse 41/IVD-80333, München, Germany.
}

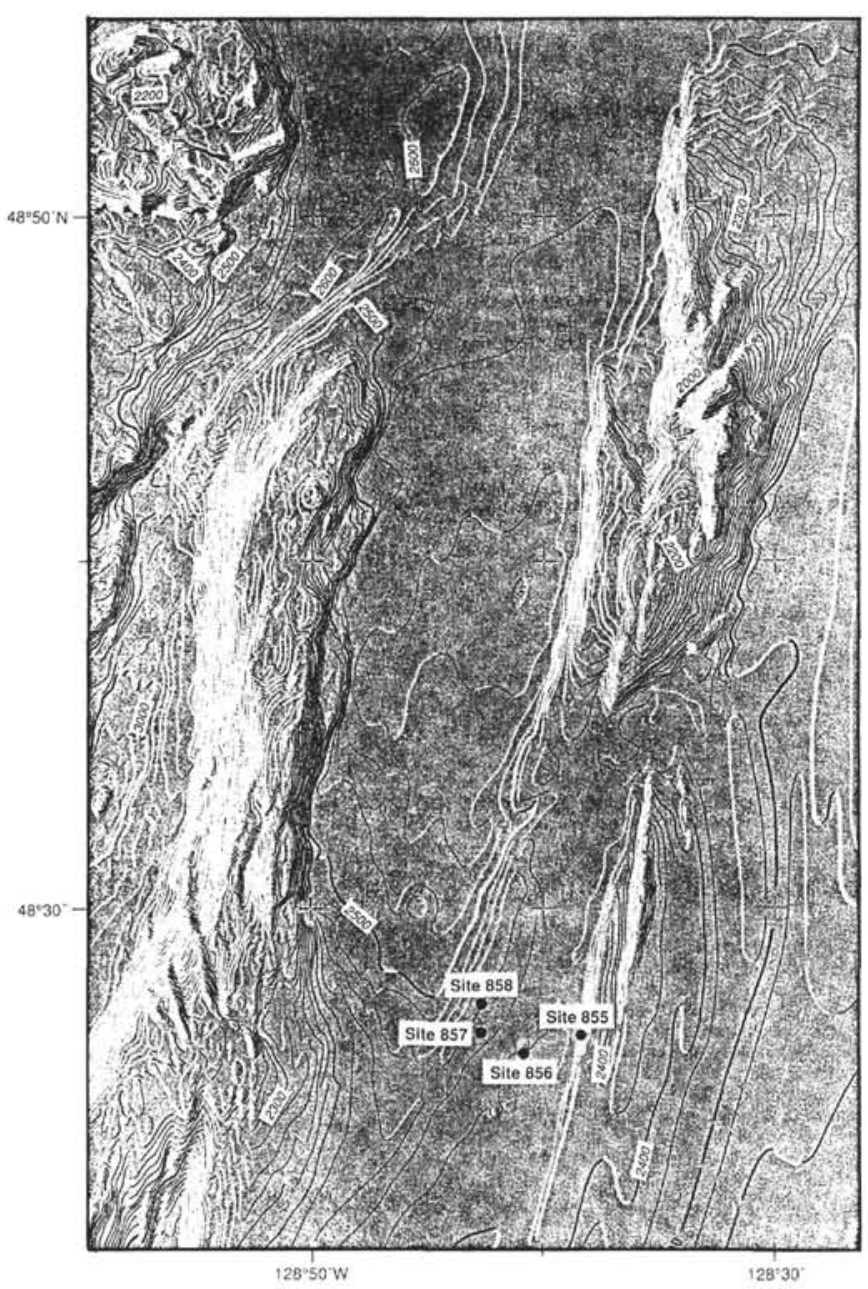

Figure 1. Location map for sites drilled in Middle Valley (Davis, Mottl, Fisher, et al., 1992).

with depth to $200^{\circ} \mathrm{C}$ at $450 \mathrm{mbsf}$. Sediment samples show an increase in the degree of alteration and silicification with increasing depth (Davis, Mottl, Fisher, et al., 1992).

Because Site 855 revealed less alteration than the others, it was a reference site for Leg 139. It was drilled just inside the scarp of the normal fault bounding the sedimented rift valley. All samples collected for paleomagnetic studies from Site 855 were slightly altered clay to silt, with a dark greenish grey color. Due to RCB coring, the 
A

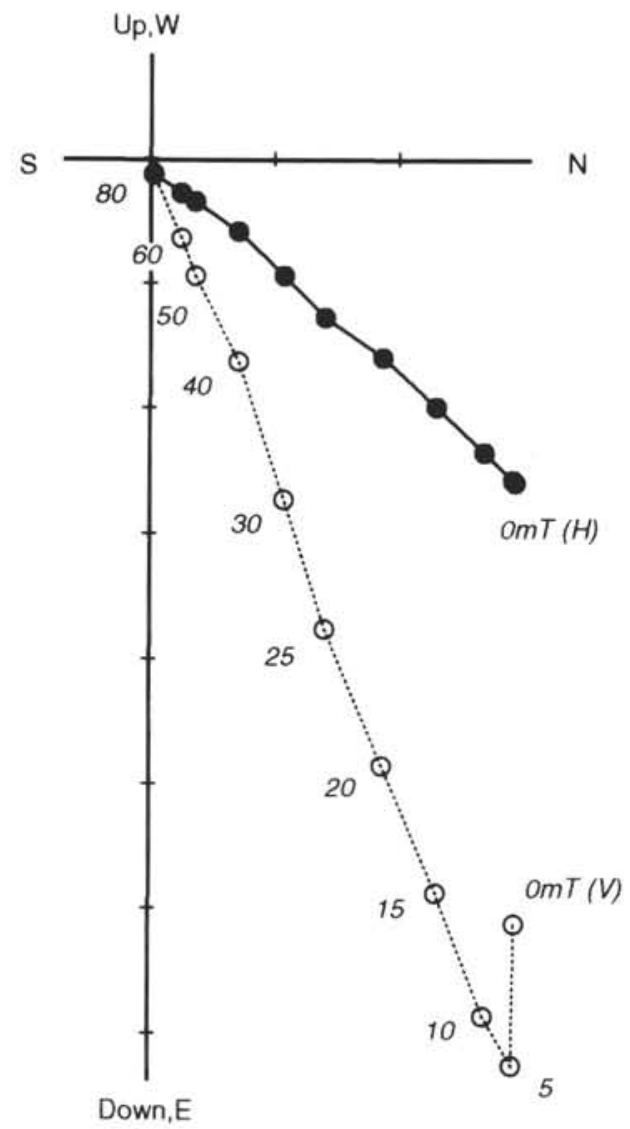

$\operatorname{div}=1 \times 10^{-7} \mathrm{Am}^{2}$

B

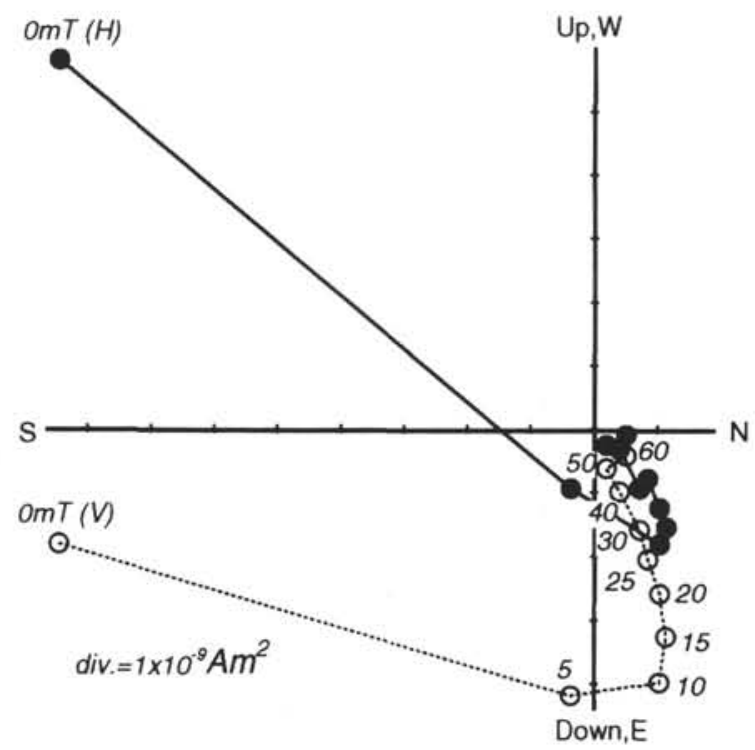

Figure 2. Zijderveld plot of progressive AF demagnetization results for two samples from Site 857. A. 139-857A-2H3, 115-117 cm. B. 139-857C-32R2, $32-34 \mathrm{~cm}$. Open circles represent the vertical component of magnetization of the samples; solid circles represent the horizontal component. unconsolidated sedimentary samples drilled at Site 855 were thoroughly disturbed; thus no reliable primary component of remanent magnetization could be obtained from these samples.

\section{METHODS}

Progressive alternating field demagnetization (PAFD) was conducted on the sediment samples to determine primary components of the remanent magnetization. Natural remanent magnetization (NRM) of the sediments was measured using a MOLSPIN portable rock magnetometer on board the Resolution, a cryogenic magnetometer at Kyoto University (SCT C112), and a LETI cryogenic magnetometer at Universität München. Alternating field demagnetization (AFD) was conducted using a Schonstedt AF demagnetizer (model 2G600) aboard the Resolution, a homemade demagnetizer with a 3-axis tumbling device at Kyoto University, and a 2G demagnetizer at Universität München. Initial susceptibility was measured with a Bartington's susceptibility meter (model M.S. 2$)$ in the low frequency mode $(0.46 \mathrm{k} \mathrm{Hz})$.

Isothermal remanent magnetization (IRM) was produced using a homemade solenoid coil and electromagnet at Kyoto University, and was measured with a spinner magnetometer (Schonstedt SSM-1A). Progressive thermal demagnetization of composite IRMs was conducted on 12 selected samples, using the method of Lowrie (1990). We produced IRMs by applying a field of $1.3 \mathrm{~T}, 0.4 \mathrm{~T}$, and $0.12 \mathrm{~T}$ in the $\mathrm{Z}$, $\mathrm{Y}$, and $\mathrm{X}$ axes, successively. After acquisition of IRMs, the samples were subjected to progressive thermal demagnetization, and the remanent magnetization was measured with a spinner magnetometer. This procedure reveals an unblocking spectrum of three different ranges of coercivity (i.e., $0-0.12 \mathrm{~T}, 0.12-0.4 \mathrm{~T}$, and $0.4-1.3 \mathrm{~T}$ ) and was helpful in identifying magnetic minerals in the samples. Thermomagnetic measurements were made with a horizontal Curie balance at Kyoto University by heating magnetic extracts from the sediment samples in Ar gas or in air with an applied magnetic field of $0.85 \mathrm{~T}$.

Hysteresis loops were obtained using a Princeton vibrating sample magnetometer at National Institute of Polar Research in Tokyo. Hysteresis parameters $(\mathrm{Js}, \mathrm{Jrs} / \mathrm{Js}, \mathrm{Hc}$, and $\mathrm{Hcr} / \mathrm{Hc}$ ) were calculated from the hysteresis loops obtained for each samples.

\section{RESULTS AND DISCUSSION NRM and Susceptibility of Sediments}

NRM intensities, stable inclinations, volume magnetic susceptibilities and hysteresis parameters (Js, Jrs/Js, Hc, and $\mathrm{Hcr} / \mathrm{Hc}$ ) of the samples from Sites 855 and 857 are listed in Table 1. As Site 855 was drilled by RCB coring and the sediment was thoroughly disturbed, paleomagnetic directions of this site might be a drilling-induced remanence. Thus, NRMs from Site 855 are not reliable in spite of stable and strong remanences.

Figure 2 shows results of progressive AFD experiments on the samples from Site 857. Stable directions were calculated using a leastsquares linear fit (Kirschvink, 1980). The secondary component of the samples, which is possibly of drilling-induced origin, increases its coercivity as depth increases (Fig. 2B). Mean stable inclination for Site 857 was calculated as $56 \pm 12^{\circ}$ by averaging stable inclinations from Holes $857 \mathrm{~A}$ and $857 \mathrm{C}$. This average inclination value is consistent with inclination expected for the axial dipole field $\left(66^{\circ}\right)$ at the site latitude $\left(48.5^{\circ} \mathrm{N}\right)$. However, we cannot conclude that these stable components of magnetization were recorded at the time of deposition.

Stable inclinations, NRM intensities, and volume magnetic susceptibilities measured for the samples from Site 857 are plotted vs. depth in Figure 3. Downcore variation of NRM intensity shows an abrupt drop at around 200-250 mbsf from $100 \mathrm{~mA} / \mathrm{m}$ to $1 \mathrm{~mA} / \mathrm{m}$. Volume magnetic susceptibility also shows an abrupt drop at this depth from $2 \times 10^{-3}$ SI to $3 \times 10^{-4} \mathrm{SI}$. 
Table 1. Magnetic properties, Sites 855 and 857.

\begin{tabular}{|c|c|c|c|c|c|c|c|c|c|}
\hline $\begin{array}{l}\text { Core, section, } \\
\text { interval }(\mathrm{cm})\end{array}$ & $\begin{array}{l}\text { Depth } \\
\text { (mbsf) }\end{array}$ & $\begin{array}{c}\text { NRM } \\
\text { intensity } \\
(\mathrm{A} / \mathrm{m})\end{array}$ & $\begin{array}{c}\text { Stable } \\
\text { inclination } \\
\text { (degrees) }\end{array}$ & $\begin{array}{c}\text { Volume } \\
\text { susceptibility } \\
\text { (SI) }\end{array}$ & $\begin{array}{c}\mathrm{Js} \\
\left(\mathrm{Am}^{2} / \mathrm{kg}\right)\end{array}$ & $\mathrm{Jrs} / \mathrm{Js}$ & $\begin{array}{l}\mathrm{Hc} \\
(\mathrm{mT})\end{array}$ & $\mathrm{Her} / \mathrm{Hc}$ & $\begin{array}{l}\text { Type of } \\
\text { composite } \\
\text { IRM } \\
\text { experiment }\end{array}$ \\
\hline \multicolumn{10}{|l|}{$139-855 \mathrm{C}-$} \\
\hline IR-3, 60-62 & 3.60 & $5.52 \mathrm{E}-02$ & & & & & & & \\
\hline IR- $4,26-28$ & 4.76 & 4.36E-02 & & $2.04 \mathrm{E}-03$ & & & & & A \\
\hline $1 \mathrm{R}-4,117-119$ & 5.67 & & & & $2.41 \mathrm{E}-01$ & 0.17 & 14.7 & 2.7 & \\
\hline $2 \mathrm{R}-3,62-64$ & 12.32 & $4.83 \mathrm{E}-02$ & & & & & & & \\
\hline $2 \mathrm{R}-4,120-122$ & 14.40 & & & & $5.03 \mathrm{E}-01$ & 0.08 & 7.8 & 3.9 & \\
\hline $3 R-5,34-36$ & 24.04 & & & & $2.14 \mathrm{E}-01$ & 0.12 & 11.1 & 3.0 & \\
\hline $4 \mathrm{R}-1,62-64$ & 27.72 & $6.37 \mathrm{E}-02$ & & & & & & & \\
\hline $4 \mathrm{R}-1,117-119$ & 28.27 & $3.79 \mathrm{E}-02$ & & & & & & & \\
\hline $4 \mathrm{R}-4,25-27$ & 31.85 & $5.06 \mathrm{E}-02$ & & $\begin{array}{l}7.85 \mathrm{E}-04 \\
1.76 \mathrm{E}-03\end{array}$ & $9.27 \mathrm{E}-02$ & 0.15 & 12.2 & 3.0 & A \\
\hline $\begin{array}{l}6 R-1,105-107 \\
6 R-2\end{array}$ & $\begin{array}{l}47.55 \\
49.38\end{array}$ & $6.19 \mathrm{E}-02$ & & $1.76 \mathrm{E}-03$ & $2.28 \mathrm{E}-01$ & 0.11 & 10.3 & 3.1 & \\
\hline $\begin{array}{l}6 \mathrm{R}-2,138-140 \\
6 \mathrm{R}-6,42-44\end{array}$ & $\begin{array}{l}49.38 \\
54.42\end{array}$ & & & & $1.83 \mathrm{E}-01$ & 0.09 & 8.4 & 4.0 & \\
\hline $7 R-6,140-142$ & 65.00 & & & & $1.88 \mathrm{E}-01$ & 0.10 & 10.0 & 3.3 & \\
\hline $10 \mathrm{R}-3,30-32$ & 88.20 & $3.14 \mathrm{E}-02$ & & $1.52 \mathrm{E}-03$ & $1.25 \mathrm{E}-01$ & 0.14 & 13.5 & 3.0 & A \\
\hline $11 \mathrm{R}-1,80-82$ & 95.40 & & & & 5.34E-01 & 0.07 & 8.1 & 3.7 & \\
\hline $139-857 \mathrm{~A}-$ & & & & & & & & & \\
\hline $1 \mathrm{H}-1,128-130$ & 3.16 & 3.35E-02 & 53 & 2.33E-03 & & & & & \\
\hline $1 \mathrm{H}-4,26-28$ & 6.66 & $7.00 \mathrm{E}-02$ & 70 & $1.60 \mathrm{E}-03$ & $1.93 \mathrm{E}-01$ & 0.13 & 11.5 & 3.1 & A \\
\hline $1 \mathrm{H}-5,110-112$ & 8.86 & $4.27 \mathrm{E}-02$ & 59 & $1.45 \mathrm{E}-03$ & & & & & \\
\hline $2 \mathrm{H}-3,35-37$ & 14.75 & $9.10 \mathrm{E}-02$ & 71 & $1.15 \mathrm{E}-03$ & & & & & \\
\hline $2 \mathrm{H}-3,115-117$ & 15.55 & $1.27 \mathrm{E}-01$ & 63 & $2.09 \mathrm{E}-03$ & 2.29E-01 & 0.10 & 10.1 & 3.5 & \\
\hline $4 \mathrm{H}-1,95-97$ & 22.85 & $2.90 \mathrm{E}-03$ & 66 & $2.60 \mathrm{E}-04$ & $8.05 \mathrm{E}-02$ & & & & \\
\hline $4 \mathrm{H}-2,42-44$ & 23.82 & $1.50 \mathrm{E}-02$ & 54 & 6.96E-04 & & 0.09 & 7.3 & 4.0 & \\
\hline $4 \mathrm{H}-2,102-104$ & 27.00 & $3.69 \mathrm{E}-02$ & 66 & $1.34 \mathrm{E}-03$ & & & & & \\
\hline $4 \mathrm{H}-7,4-6$ & 30.23 & $1.97 \mathrm{E}-02$ & 46 & $8.13 \mathrm{E}-04$ & & & & & \\
\hline $5 \mathrm{H}-3,100-102$ & 35.15 & $1.60 \mathrm{E}-02$ & 81 & 7.72E-04 & $7.24 \mathrm{E}-02$ & & & & \\
\hline $5 \mathrm{H}-4,20-22$ & 36.10 & $2.30 \mathrm{E}-02$ & 66 & $6.48 \mathrm{E}-04$ & & 0.13 & 9.7 & 3.3 & \\
\hline $5 \mathrm{H}-6,111-113$ & 39.50 & $9.86 \mathrm{E}-03$ & 74 & $\begin{array}{l}1.33 \mathrm{E}-03 \\
1.31 \mathrm{E}-03\end{array}$ & $1.23 \mathrm{E}-01$ & 0.10 & & & \\
\hline $6 \mathrm{H}-3,20-22$ & 44.10 & $9.20 \mathrm{E}-02$ & 60 & $\begin{array}{l}1.31 \mathrm{E}-03 \\
2.43 \mathrm{E}-03\end{array}$ & $\begin{array}{l}1.23 \mathrm{E}-01 \\
2.18 \mathrm{E}-01\end{array}$ & 0.12 & 9.5 & 3.4 & \\
\hline $7 \mathrm{H}-2,77-79$ & 51.49 & $4.55 \mathrm{E}-01$ & 50 & $2.43 \mathrm{E}-03$ & & 0.12 & 11.3 & 3.1 & \\
\hline $7 \mathrm{H}-3,58-60$ & 52.80 & $2.01 \mathrm{E}-01$ & 47 & $1.54 \mathrm{E}-03$ & & & & & \\
\hline $8 \mathrm{H}-4,127-129$ & 65.67 & 1.17E-01 & 65 & $1.81 \mathrm{E}-03$ & & & & & \\
\hline $8 \mathrm{H}-4,130-132$ & 65.70 & $6.10 \mathrm{E}-02$ & 60 & $2.00 \mathrm{E}-03$ & & & & & \\
\hline $8 \mathrm{H}-5,8-10$ & 65.98 & 1.38E-01 & 57 & $1.57 \mathrm{E}-03$ & $\begin{array}{l}1.59 \mathrm{E}-01 \\
4.05 \mathrm{E}-01\end{array}$ & 0.12 & 10.9 & 3.0 & \\
\hline $9 \mathrm{H}-4,56-58$ & 74.46 & $9.80 \mathrm{E}-02$ & 62 & 4.86E-03 & $\begin{array}{l}\text { 4.05E-01 } \\
2.46 \mathrm{E}-01\end{array}$ & 0.09 & 8.9 & 3.4 & A \\
\hline $10 \mathrm{H}-3,31-33$ & 82.21 & $4.00 \mathrm{E}-02$ & 54 & $3.14 \mathrm{E}-03$ & $2.46 \mathrm{E}-01$ & 0.11 & 9.8 & 3.2 & \\
\hline $13 X-1,127-129$ & 102.77 & $1.52 \mathrm{E}-01$ & 68 & $1.65 \mathrm{E}-03$ & $1.60 \mathrm{E}-01$ & 0.09 & 9.3 & 3.4 & \\
\hline $13 X-3,130-132$ & 105.80 & $7.30 \mathrm{E}-02$ & 65 & $1.71 \mathrm{E}-03$ & & & & & \\
\hline $13 X-5,81-83$ & 108.31 & $5.06 \mathrm{E}-02$ & 67 & $1.98 \mathrm{E}-03$ & $1.72 \mathrm{E}-01$ & 0.08 & 8.2 & 3.5 & \\
\hline 139-857C- & & & & & & & & & \\
\hline $6 \mathrm{R}-2,11-13$ & 87.81 & $5.20 \mathrm{E}-02$ & $\begin{array}{l}26 \\
83\end{array}$ & $1.96 \mathrm{E}-03$ & $\begin{array}{l}2.16 \mathrm{E}-01 \\
5.68 \mathrm{E}-02\end{array}$ & 0.10 & 9.7 & $\begin{array}{l}3.4 \\
3.4\end{array}$ & \\
\hline $10 \mathrm{R}-1,45-47$ & 124.55 & $3.66 \mathrm{E}-02$ & 83 & 7.45E-04 & $\begin{array}{l}5.68 \mathrm{E}-02 \\
2.00 \mathrm{E}-01\end{array}$ & 0.13 & 9.6 & & \\
\hline $12 \mathrm{R}-2,80-82$ & 145.80 & $1.67 \mathrm{E}-01$ & 29 & $2.09 \mathrm{E}-03$ & & 0.08 & 8.2 & 3.7 & \\
\hline $14 \mathrm{R}-2,125-127$ & 165.55 & $7.50 \mathrm{E}-01$ & 47 & $6.04 \mathrm{E}-03$ & $3.29 \mathrm{E}-01$ & 0.06 & 6.4 & 4.0 & \\
\hline $17 \mathrm{R}-1,67-69$ & 192.57 & $1.10 \mathrm{E}-02$ & 41 & $1.11 \mathrm{E}-03$ & & & & & \\
\hline $19 \mathrm{R}-1,126-128$ & 212.56 & $2.00 \mathrm{E}-03$ & 64 & $6.15 \mathrm{E}-04$ & $3.28 \mathrm{E}-02$ & 0.04 & 3.6 & 3.4 & A \\
\hline $21 \mathrm{R}-1,23-25$ & 230.83 & $1.55 \mathrm{E}-01$ & 55 & $2.12 \mathrm{E}-03$ & $1.61 \mathrm{E}-01$ & 0.05 & 5.2 & 4.4 & A \\
\hline $21 R-2,35-37$ & 232.45 & $2.00 \mathrm{E}-02$ & 40 & $1.66 \mathrm{E}-03$ & & & & & \\
\hline $24 \mathrm{R}-1,12-14$ & 259.33 & $5.00 \mathrm{E}-04$ & 60 & $3.22 \mathrm{E}-04$ & & & & & \\
\hline $24 \mathrm{R}-1,103-105$ & 260.23 & $7.15 \mathrm{E}-04$ & 51 & $2.92 \mathrm{E}-04$ & $1.32 \mathrm{E}-02$ & 0.21 & 10.7 & 2.9 & \\
\hline $25 \mathrm{R}-1.55-57$ & 269.35 & 4.10E-04 & 30 & 3.13E-04 & & & & & \\
\hline $26 \mathrm{R}-1,11-13$ & 274.61 & $1.60 \mathrm{E}-03$ & 59 & $3.08 \mathrm{E}-04$ & $1.09 \mathrm{E}-02$ & 0.34 & 11.3 & 2.7 & \\
\hline $26 \mathrm{R}-1,41-43$ & 274.91 & $4.90 \mathrm{E}-04$ & 57 & $3.18 \mathrm{E}-04$ & & & & & \\
\hline $27 \mathrm{R}-1,45-47$ & 285.56 & 4.63E-04 & 54 & $3.24 \mathrm{E}-04$ & & & & & \\
\hline $28 \mathrm{R}-1,131-133$ & 296.61 & $1.29 \mathrm{E}-03$ & 65 & $3.10 \mathrm{E}-04$ & & & & & $\mathrm{~B}$ \\
\hline $28 \mathrm{R}-2,47-49$ & 295.77 & 1.13E-03 & 55 & 2.92E-04 & $3.16 \mathrm{E}-02$ & 0.17 & 11.0 & 2.8 & B \\
\hline $29 \mathrm{R}-2,38-40$ & 305.28 & 5.60E-04 & 50 & 3.21E-04 & & & 102 & 24 & \\
\hline $30 \mathrm{R}-2,21-23$ & 314.81 & $5.76 \mathrm{E}-03$ & 42 & $2.27 \mathrm{E}-04$ & $5.64 \mathrm{E}-02$ & 0.15 & 10.2 & & \\
\hline 30R-1, 106-108 & 314.16 & $6.70 \mathrm{E}-04$ & 61 & 2.81E-04 & & & & & \\
\hline & $\begin{array}{l}323.77 \\
328.79\end{array}$ & $\begin{array}{l}4.70 \mathrm{E}-04 \\
6.70 \mathrm{E}-04\end{array}$ & $\begin{array}{l}62 \\
49\end{array}$ & $\begin{array}{l}3.34 \mathrm{E}-04 \\
3.17 \mathrm{E}-04\end{array}$ & & & & & \\
\hline $\begin{array}{l}32 \mathrm{R}-1,119-121 \\
32 \mathrm{R}-2,34-36\end{array}$ & $\begin{array}{l}328.79 \\
329.44\end{array}$ & $\begin{array}{l}0.70 \mathrm{E}-04 \\
1.74 \mathrm{E}-03\end{array}$ & 58 & $2.75 \mathrm{E}-04$ & $2.61 \mathrm{E}-02$ & 0.22 & 13.1 & 2.2 & \\
\hline $32 \mathrm{R}-2,125-127$ & 330.35 & $4.20 \mathrm{E}-04$ & 57 & $3.23 \mathrm{E}-04$ & & & & & \\
\hline $33 \mathrm{R}-1,96-98$ & 333.34 & $4.20 \mathrm{E}-04$ & 57 & $3.31 \mathrm{E}-04$ & & & & & \\
\hline $34 \mathrm{R}-1,124-126$ & 337.73 & $4.70 \mathrm{E}-04$ & 59 & $2.33 \mathrm{E}-04$ & & & & & \\
\hline $35 \mathrm{R}-2,26-28$ & 343.06 & $5.10 \mathrm{E}-04$ & 47 & $2.13 \mathrm{E}-04$ & & & & & \\
\hline $36 \mathrm{R}-1,136-138$ & 347.46 & $4.70 \mathrm{E}-04$ & 51 & 4.35E-04 & & & & & \\
\hline $36 \mathrm{R}-2,22-24$ & 347.82 & $1.40 \mathrm{E}-03$ & 51 & $2.80 \mathrm{E}-04$ & & & & & B \\
\hline $37 \mathrm{R}-1,108-110$ & 351.88 & $6.30 \mathrm{E}-04$ & 55 & $4.00 \mathrm{E}-04$ & & & & & \\
\hline $38 \mathrm{R}-1,30-32$ & 356.10 & $1.74 \mathrm{E}-03$ & & 2.92E-04 & $2.08 \mathrm{E}-02$ & 0.26 & 11.8 & 2.2 & \\
\hline $38 \mathrm{R}-2,44-46$ & 357.74 & $1.70 \mathrm{E}-04$ & 57 & $3.57 \mathrm{E}-04$ & & & & & \\
\hline $40 \mathrm{R}-1,67-69$ & 366.07 & $2.00 \mathrm{E}-04$ & 56 & $2.89 \mathrm{E}-04$ & & & & & \\
\hline $42 \mathrm{R}-1,110-112$ & 380.80 & $2.40 \mathrm{E}-04$ & 63 & $3.34 \mathrm{E}-04$ & & & & & \\
\hline $42 \mathrm{R}-2,112-114$ & 382.32 & 3.66E-03 & 32 & 2.92E-04 & $8.66 \mathrm{E}-02$ & 0.14 & 11.3 & 2.2 & B \\
\hline $44 \mathrm{R}-2,29-31$ & 390.61 & $2.70 \mathrm{E}-04$ & 58 & 3.36E-04 & & & & & \\
\hline $45 \mathrm{R}-1,18-20$ & 394.48 & $1.05 \mathrm{E}-03$ & 73 & 3.08E-04 & 4.45E-02 & 0.22 & 13.9 & 2.0 & \\
\hline $46 \mathrm{R}-1,89-91$ & 399.89 & $4.00 \mathrm{E}-04$ & 69 & $3.41 \mathrm{E}-04$ & & & & & \\
\hline $48 \mathrm{R}-1,10-12$ & 408.80 & $3.60 \mathrm{E}-04$ & 65 & 3.83E-04 & & & & & \\
\hline $48 R-2,24-26$ & 410.44 & $9.00 \mathrm{E}-04$ & 66 & 2.59E-04 & $1.63 \mathrm{E}-01$ & 0.11 & 10.7 & 2.5 & \\
\hline $49 R-2,92-94$ & 416.12 & $1.73 \mathrm{E}-03$ & 33 & 3.08E-04 & $8.40 \mathrm{E}-02$ & 0.14 & 11.3 & 2.3 & \\
\hline $50 \mathrm{R}-2,15-17$ & 419.95 & $1.92 \mathrm{E}-03$ & 37 & 3.30E-04 & & & & & B \\
\hline $51 R-1,108-110$ & 424.48 & $2.62 \mathrm{E}-03$ & & $3.45 \mathrm{E}-04$ & & & & & \\
\hline $52 \mathrm{R}-1,141-143$ & 429.21 & $3.14 \mathrm{E}-03$ & 50 & $3.07 \mathrm{E}-04$ & & & & & \\
\hline $53 \mathrm{R}-1,60-62$ & 433.40 & $2.80 \mathrm{E}-02$ & & $2.60 \mathrm{E}-04$ & & & & & \\
\hline $56 \mathrm{R}-1,22-24$ & 446.72 & $1.74 \mathrm{E}-03$ & & $3.16 \mathrm{E}-04$ & & & & & B \\
\hline $66 \mathrm{R}-1,12-14$ & 538.92 & $3.90 \mathrm{E}-04$ & 64 & $2.50 \mathrm{E}-04$ & & & & & \\
\hline
\end{tabular}




\section{Curie Temperatures and Unblocking Temperatures}

The results of the composite IRM experiments (Lowrie, 1990) on Sites 855 and 857 are plotted in the left column of Figures 4 and 5 . Susceptibilities measured just after each heating step were also monitored and are plotted in the right column of Figures 4 and 5. The results can be roughly classified into two types: type A for Site 855 samples and Site 857 samples from seafloor to $250 \mathrm{mbsf}$, and type B for Site 857 samples below 250 mbsf (Table 1). Soft $(0-0.12 \mathrm{~T})$ and medium (0.12-0.4T) components of type A samples have two unblocking temperatures of about $300^{\circ} \mathrm{C}$ and $570^{\circ} \mathrm{C}$. Soft $(0-0.12 \mathrm{~T})$ and medium $(0.12-0.4 \mathrm{~T})$ components of type B show single unblocking temperature of about $570^{\circ} \mathrm{C}$. The susceptibility of type A samples monitored during heating shows abrupt decreases at about $300^{\circ} \mathrm{C}$, and those of type B samples show increases at $400^{\circ} \mathrm{C}$.

The results of thermomagnetic analysis for magnetic extracts of type A samples indicate that the Curie temperature of the magnetic mineral is about $580^{\circ} \mathrm{C}$ (Fig. 6). The thermomagnetic curve also shows a kink at $360^{\circ} \mathrm{C}$ in air and at $450^{\circ} \mathrm{C}$ in $\mathrm{Ar}$ atmosphere.

\section{Hysteresis Parameters}

We conducted IRM acquisition experiments on the samples; IRM acquisition curves reached saturation at about $0.2 \mathrm{~T}$ (Fig. 7).

Hysteresis parameters were measured on the powder samples of the sediments. The results of hysteresis measurement for Site 855 are plotted in Figure 8. These plots show no systematic variation with depth. Js is about $0.2 \mathrm{Am}^{2} / \mathrm{kg}, \mathrm{Jrs} / \mathrm{Js}$ is about $0.1, \mathrm{Hc}$ is about $10 \mathrm{mT}$, and $\mathrm{Hcr} / \mathrm{Hc}$ is about 3 .

The hysteresis parameters for Site 857 samples are plotted in Figure 9. Js fluctuates significantly; values are about $0.2 \mathrm{Am}^{2} / \mathrm{kg}$ above $250 \mathrm{mbsf}$ and below this depth drop to less than $0.1 \mathrm{Am}^{2} / \mathrm{kg}$. $\mathrm{Jrs} / \mathrm{Js}$ values decrease gradually from 0.1 at sea bottom to 0.05 at 250 mbsf. Jrs/Js abruptly increases to 0.2 at $250 \mathrm{mbsf}$ and remains constant below. Hc is about $9 \mathrm{mT}$ above $200 \mathrm{mbsf}$, reaches minimum at 200-250 mbsf, and remains constant about $11 \mathrm{mT}$ below $250 \mathrm{mbsf}$. $\mathrm{Hcr} / \mathrm{Hc}$ is about 3.5 above $250 \mathrm{mbsf}$ and below $250 \mathrm{mbsf}$ decreases to about 2.5. It is remarkable that all the hysteresis parameters for Site 857 change at $250 \mathrm{mbsf}$.

\section{ACKNOWLEDGMENTS}

We would like to thank Minoru Funaki for the use of a vibrating sample magnetometer at National Institute of Polar Research in Tokyo. We extend our hearty thanks to $\mathrm{M}$. Torii for reviewing the manuscript.

\section{REFERENCES *}

Channell, J.E.T., and Hawthorne, T., 1990. Progressive dissolution of titanomagnetites at ODP Site 653 (Tyrrhenian Sea). Earth Planet. Sci. Lett., 96:469-480.

Davis, E.E., Mottl, M.J., Fisher, A.T., et al., 1992. Proc. ODP, Init. Repts., 139: College Station, TX (Ocean Drilling Program).

Karlin, R., 1990. Magnetite diagenesis in marine sediments from the Oregon continental margin. J. Geophys. Res., 95:4405-4419.

King, J.W., and Channell, J.E.T., 1991. Sedimentary magnetism, environmental magnetism, and magnetostratigraphy. Rev. Geophys., Suppl., 358-370.

Kirschvink, J.L., 1980. The least-squares line and plane and analysis of palaeomagnetic data. Geophys. J. R. Astron. Soc., 62:699-718.

Lowrie, W., 1990. Identification of ferromagnetic minerals in a rock by coercivity and unblocking temperature properties. Geophys. Res. Lett., 17:159-162.

Torii, M., Hayashida, A., Vigliotti, L., and Wippern, J., 1992. Rock magnetic properties of sediments from Site 797, Japan Sea. In Tamaki, K., Suyehiro, K., Allan, J., McWilliams, M., et al., Proc. ODP, Sci. Results, 127/128 (Pt. 2): College Station, TX (Ocean Drilling Program), 947-957.

- Abbreviations for names of organizations and publications in ODP reference lists follow the style given in Chemical Abstracts Service Source Index (published by American Chemical Society).

Date of initial receipt: 22 March 1993

Date of acceptance: 30 July 1993

Ms 139SR-244 

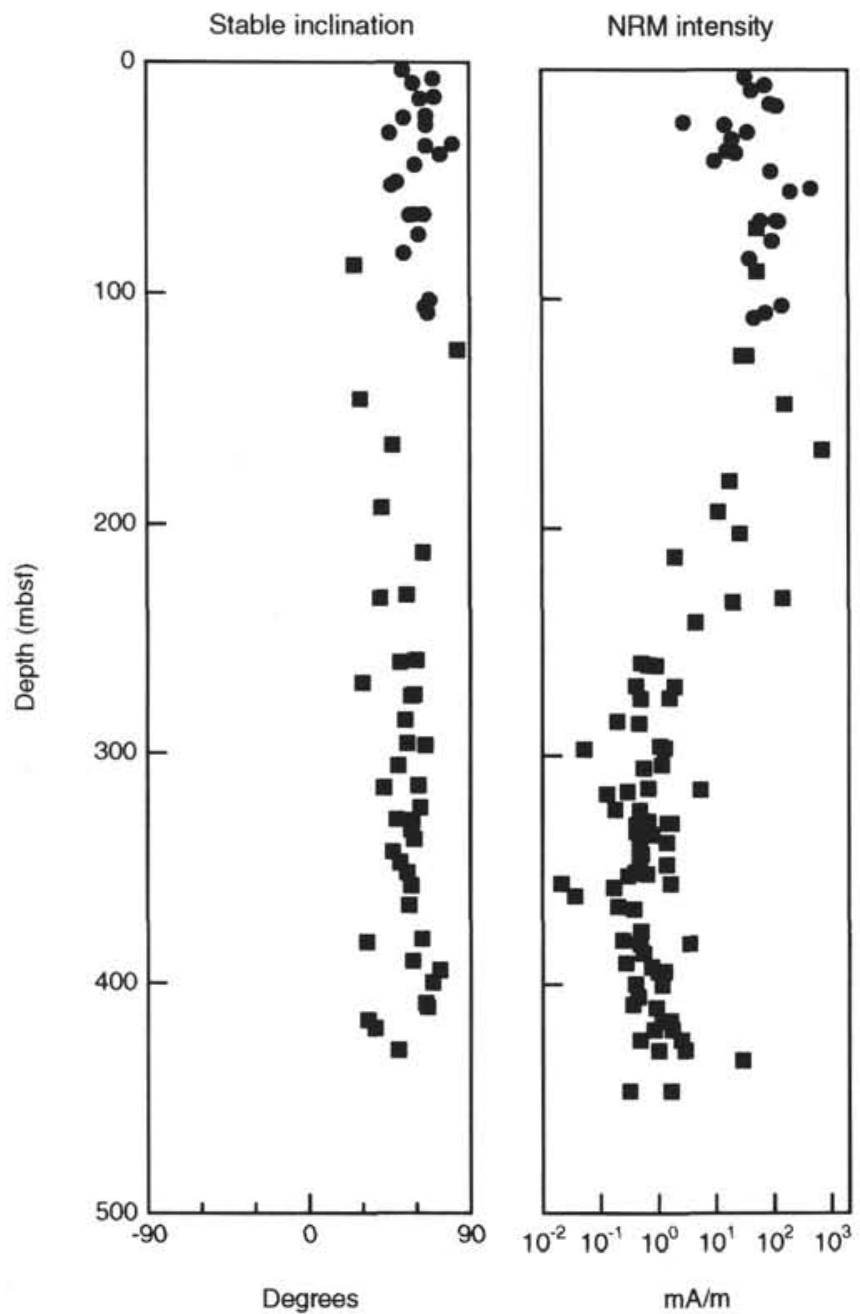

Volume susceptibility

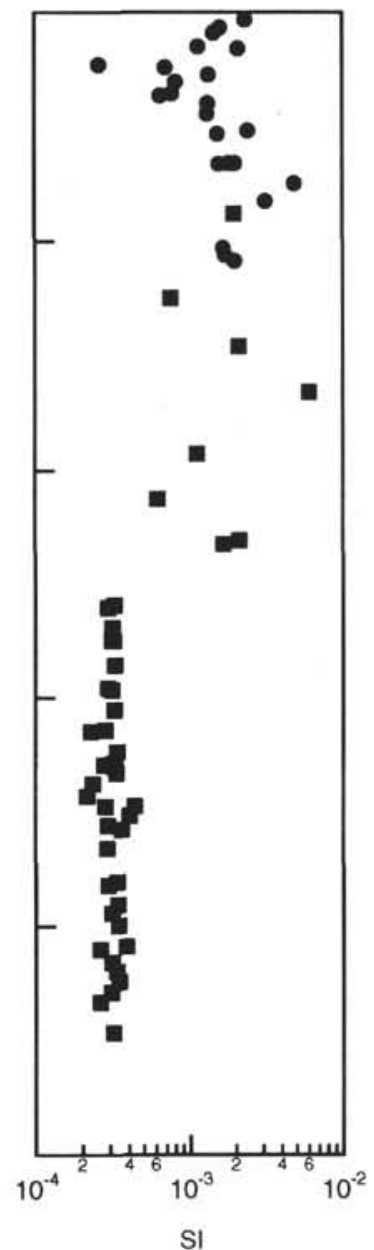

Figure 3. Plots of stable inclination, NRM intensity, and volume magnetic susceptibility vs. depth for Site 857 . Solid circles and squares represent results from Hole $857 \mathrm{~A}$ and Hole $857 \mathrm{C}$, respectively. 

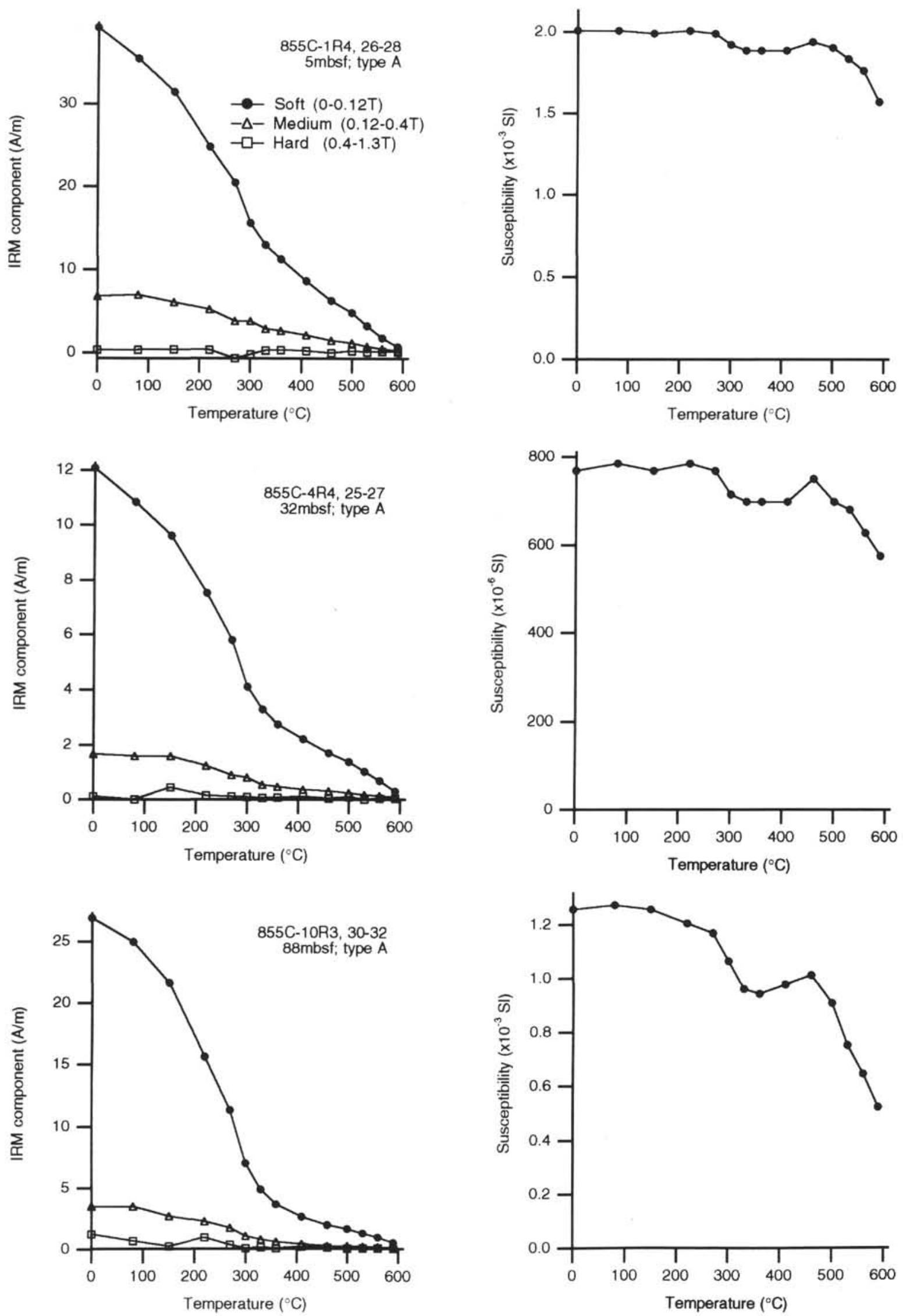

Figure 4. Composite IRM plots (left column) and susceptibility after each heating step (right column) for Site 855. 

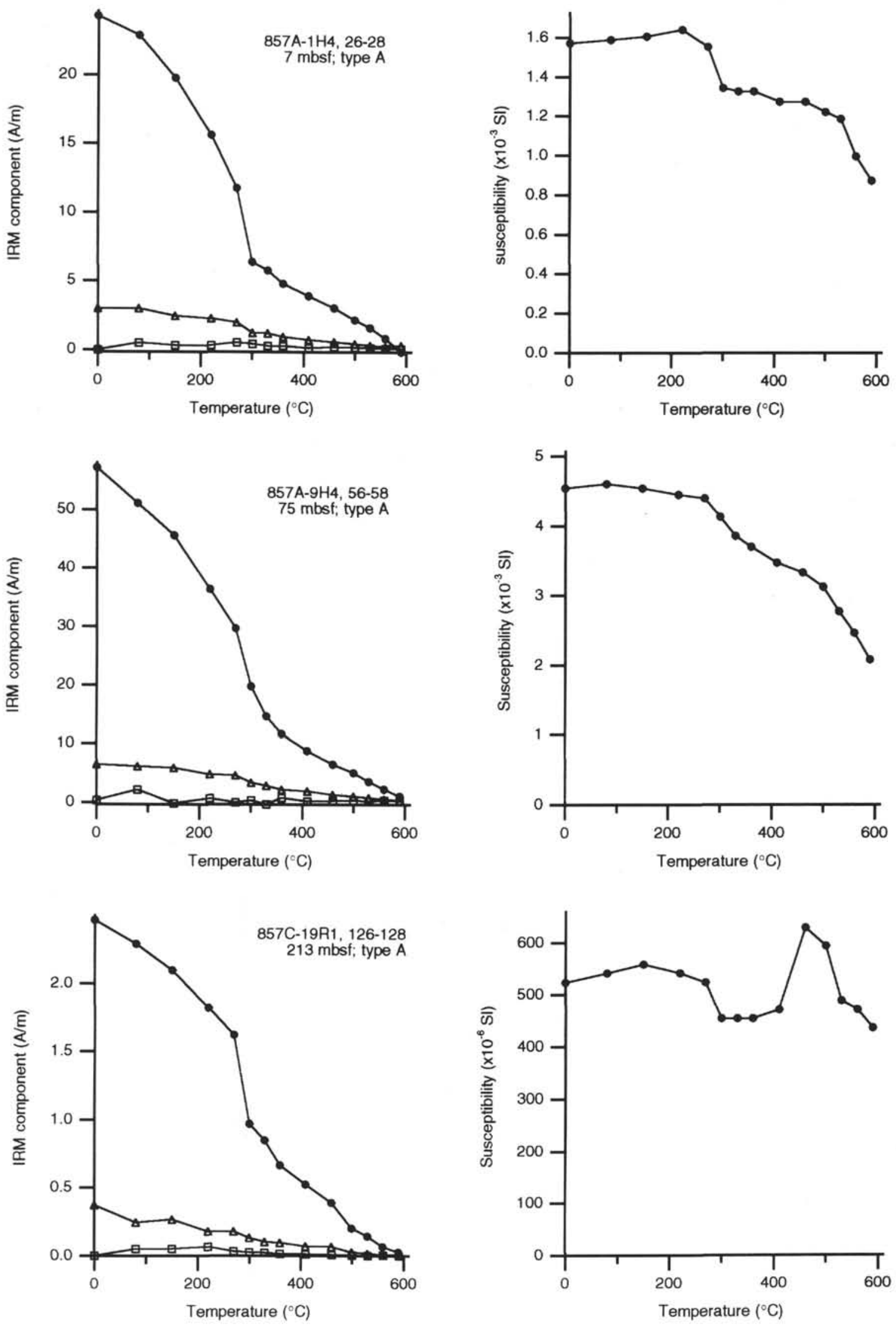

Figure 5. Composite IRM plots and susceptibility after each heating step for Site 857. Symbols used are as in Figure 4. 

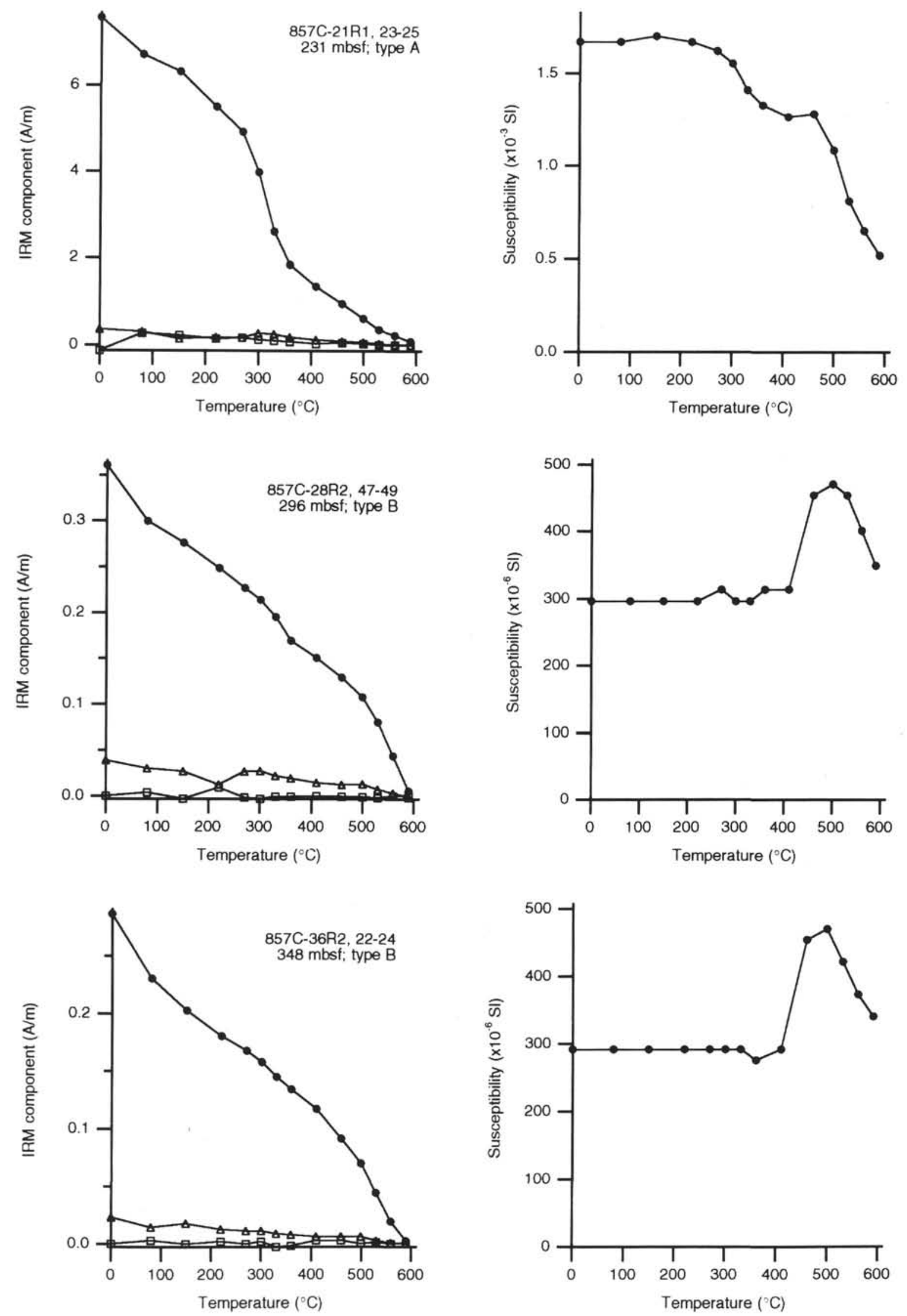

Figure 5 (continued). 

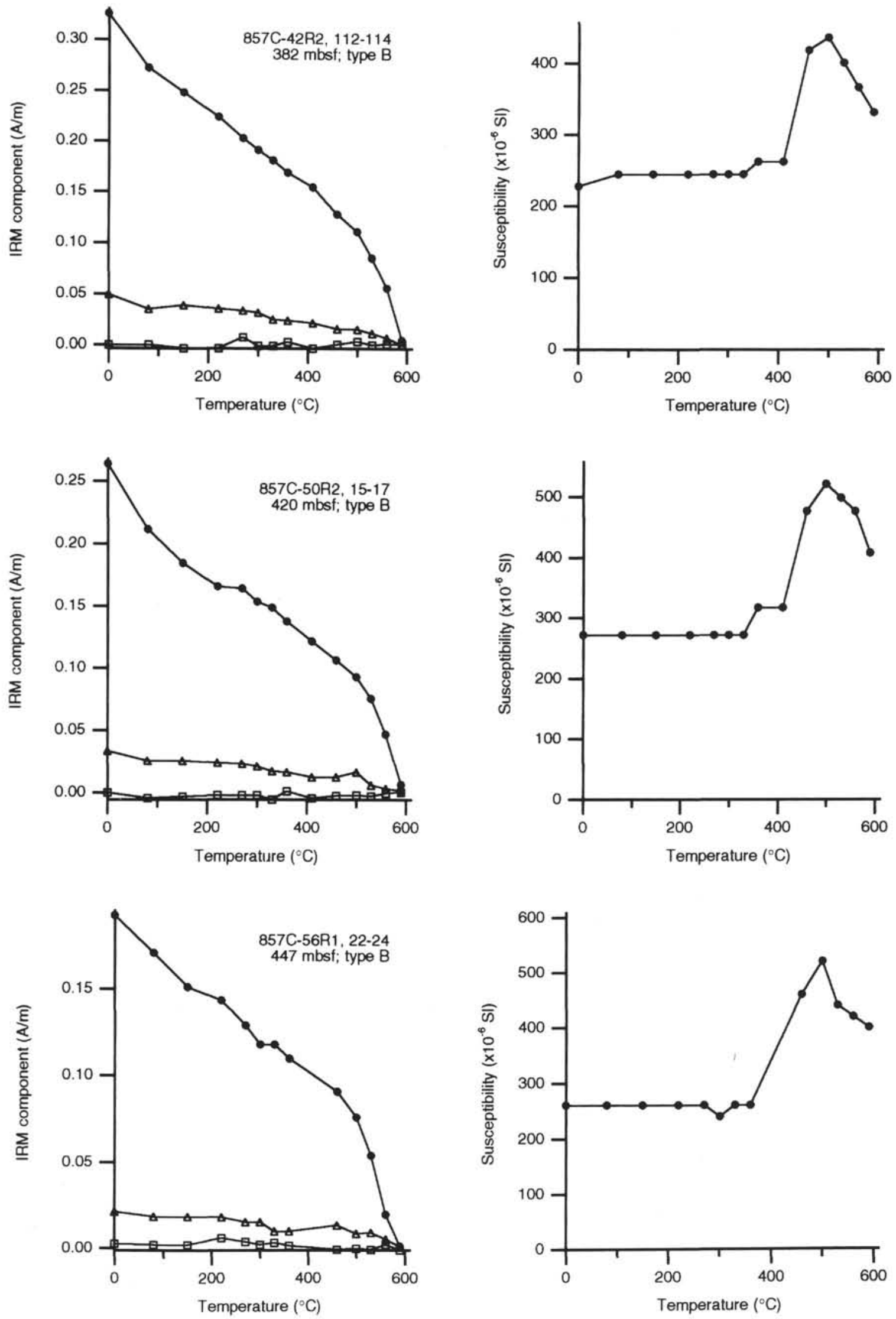

Figure 5 (continued). 

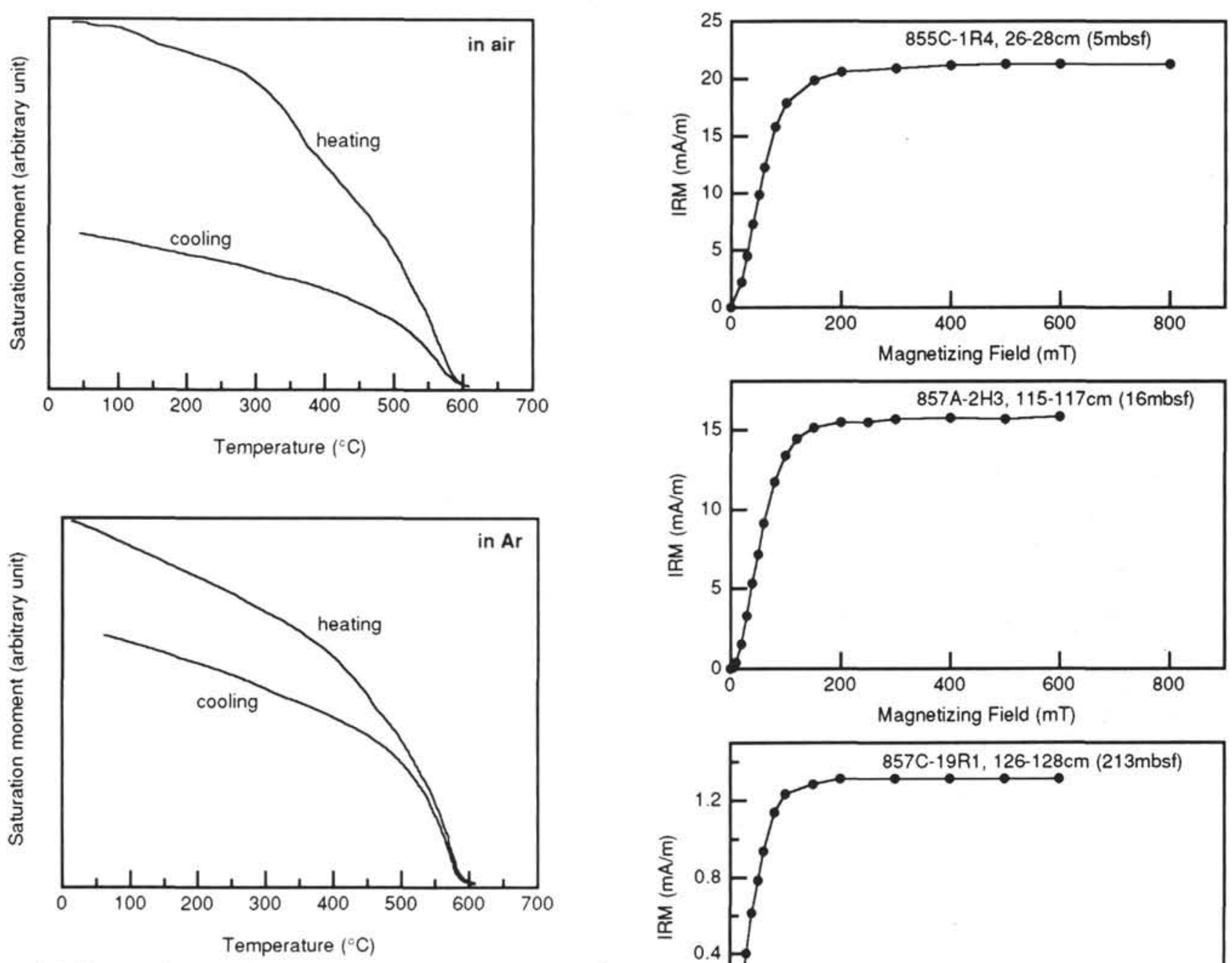

Figure 6. Js-T curves for magnetic separates of Sample 139-857A-2H3, $115-117 \mathrm{~cm}$ in air and Ar.
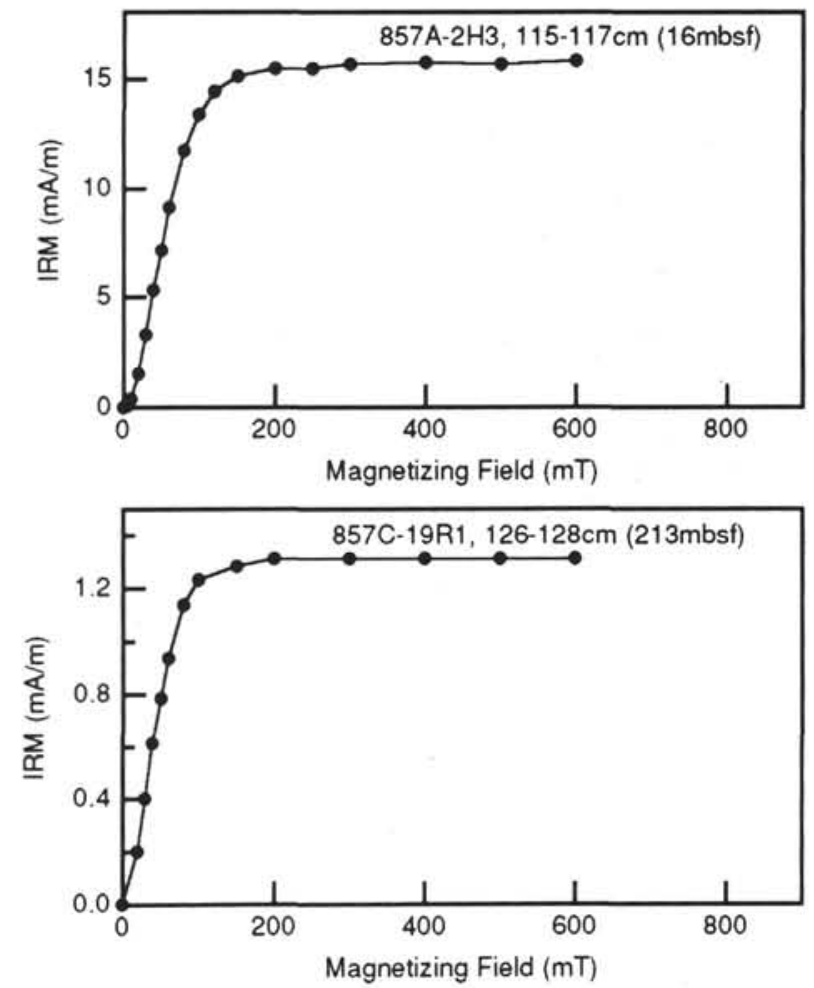

Figure 7. IRM acquisition curves for samples indicated. 


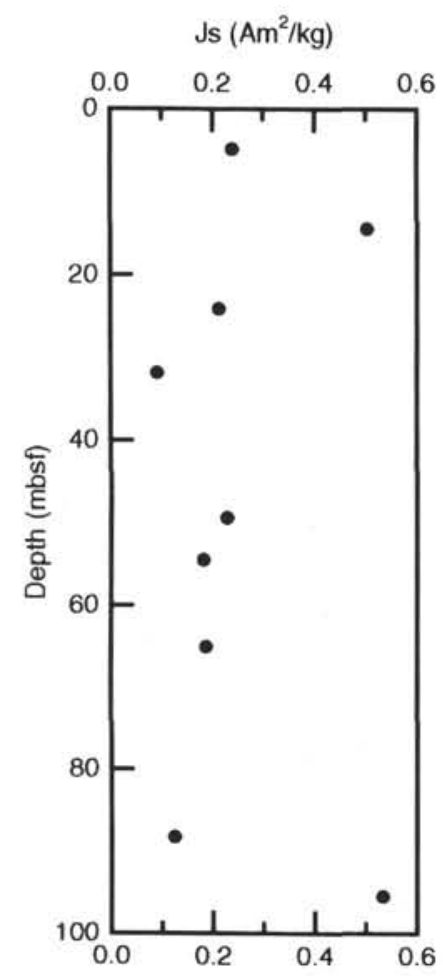

$\mathrm{Hc}(\mathrm{mT})$

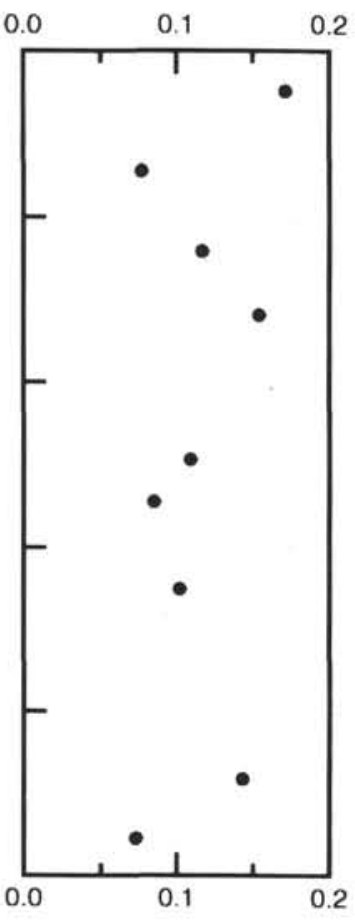

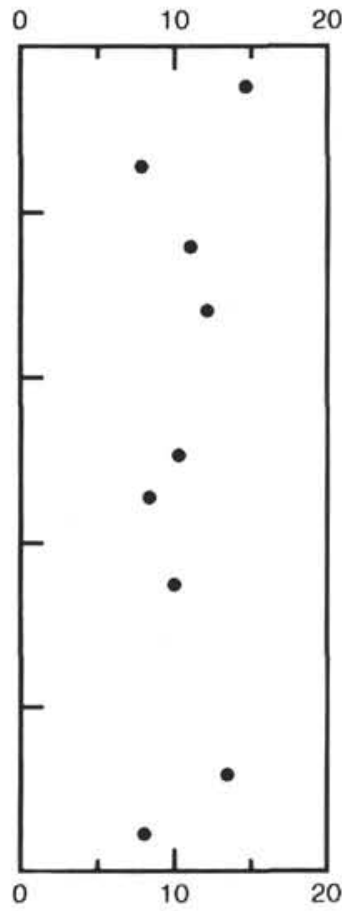

$\mathrm{Hcr} / \mathrm{Hc}$

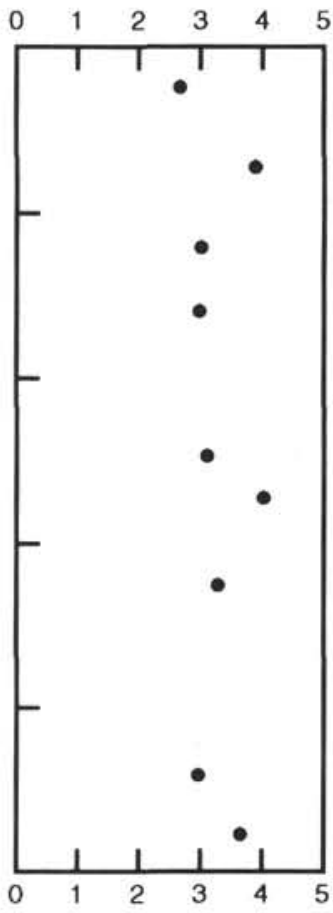

Figure 8. Hysteresis parameters (Js, Jrs/Js, Hc, and $\mathrm{Hcr} / \mathrm{Hc}$ ) for Hole 855C, plotted vs. depth.
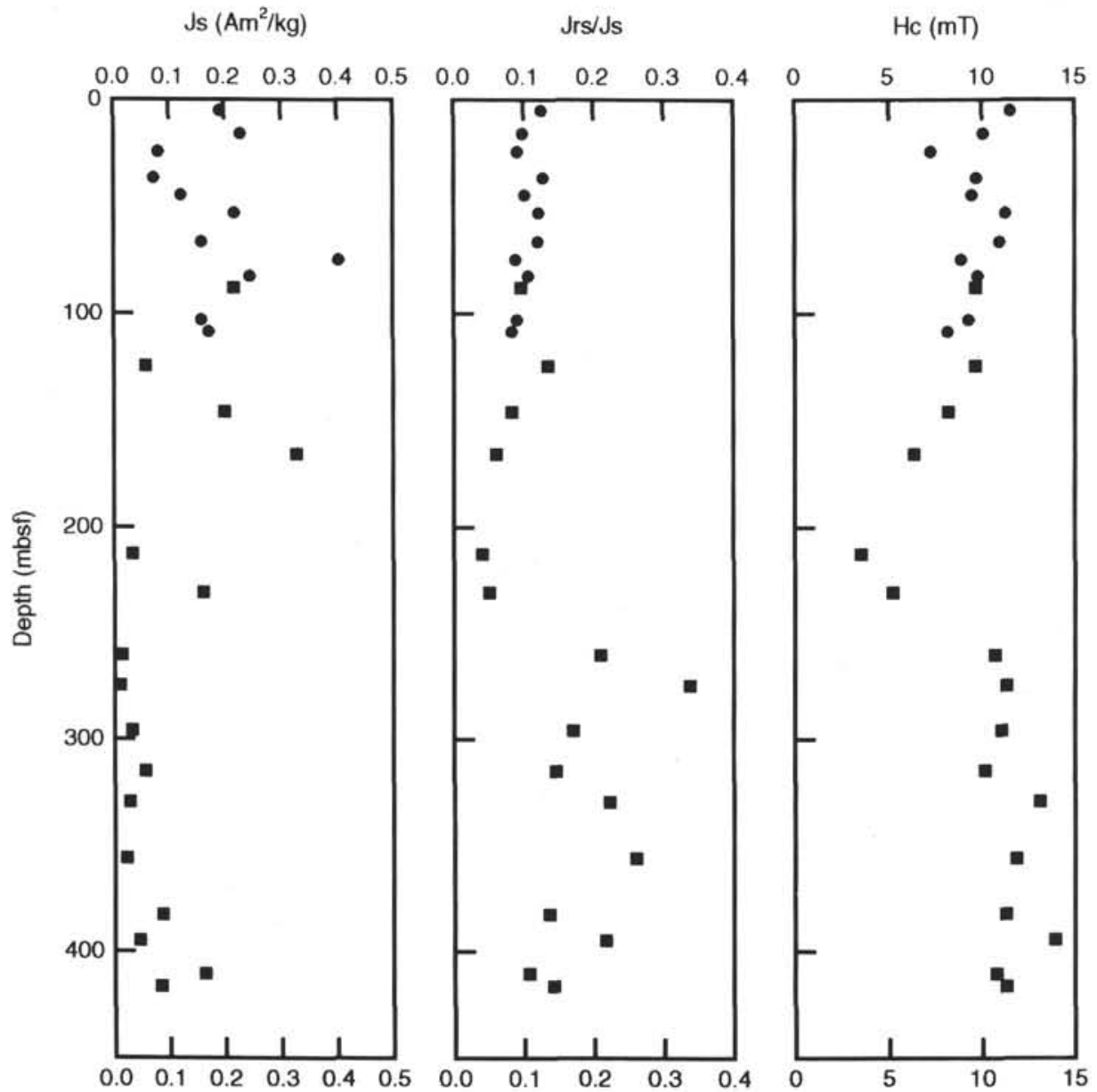

$\mathrm{Hcr} / \mathrm{Hc}$

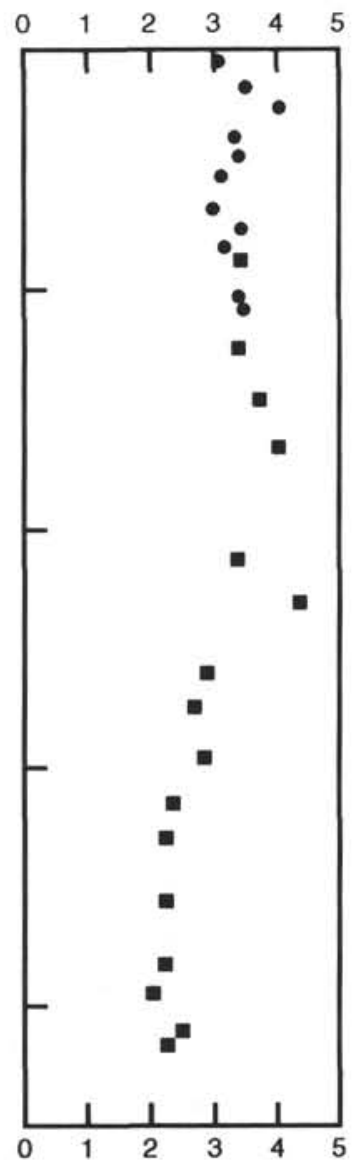

Figure 9. Hysteresis parameters ( $\mathrm{Js}, \mathrm{Jrs} / \mathrm{Js}, \mathrm{Hc}$, and $\mathrm{Hcr} / \mathrm{Hc}$ ) for Site 857, plotted vs. depth. 